Universitas, Volumen 1, Año 1, 2007, 33-38

2007 UNAN-León, Editorial Universitaria

\title{
Puntos críticos en la evaluación de impacto ambiental de la Camaronicultura en el Pacífico de Nicaragua, durante su proceso productivo: Producción de larvas, operación y abandono de Granjas.
}

\author{
Jorge Isaac Flores ${ }^{1}$, Evenor Martínez González², Pedrarias Dávila Prado ${ }^{1 *}$ \\ ${ }^{1}$ Gabinete de Ecología y Medio Ambiente, Facultad de Ciencias, Universidad Nacional Autónoma de Nicaragua, León. \\ (UNAN-León). \\ ${ }^{2}$ Laboratorio Investigaciones Marinas y Acuícolas, Facultad de Ciencias, Universidad Nacional Autónoma de Nicaragua, León. \\ (UNAN-León).
}

RESUMEN

\begin{abstract}
La camaronicultura es un sector productivo en aumento con un gran potencial, especialmente en la costa del Pacifico de Nicaragua. Actualmente se discute sobre sus posibles impactos ambientales. Este articulo trata, de identificar y caracterizar los principales impactos ambientales de su proceso productivo que ayude a señalar aquellos puntos críticos para la evaluación de impacto ambiental. Con este objetivo se visitaron tres empresas implicadas, en cada una de las etapas del proceso productivo: producción de larvas, operación y abandono de granjas camaroneras respectivamente, donde se realizaron entrevistas semi-estructuradas y observación directa en el campo. Uno de los principales puntos críticos identificados en la evaluación de impactos ambientales durante la operación de granjas camaroneras y la producción de larvas, resultó ser la contaminación del agua por descargas en cada ciclo productivo. Este posible impacto podría caracterizarse como un impacto directo, acumulativo y posiblemente irrecuperable sobre el medio acuático, con consecuencias significativas sobre el medio social, que a su vez es receptor de impactos derivados del abandono de granjas camaroneras al crear conflictos legales sobre la tenencia de la tierra.
\end{abstract}

Palabras Claves: Estudio de impacto ambiental, Identificación de impactos, Caracterización de impactos, Contaminación del agua, Granjas camaroneras.

\section{INTRODUCCIÓN}

A nivel mundial, la camaronicultura es un sector productivo en aumento. La fuerte demanda del mercado internacional ha elevado la producción mundial, para el año 2001 se registraba una producción de tres millones de toneladas métricas por un valor aproximado de 12,000 millones de dólares ${ }^{[9]}$. Según PND (2003) para ese año, el $4 \%$ de las exportaciones de Nicaragua fueron de camarón de cultivo, superando a rubros tradicionales como el tabaco, el banano, ajonjolí y plata, lo que ilustra la importancia económica de este sector para el país.

Por otro lado, Nicaragua posee un gran potencial para el desarrollo del sector, en especial la costa del Pacifico. Según Ortega ${ }^{[8]}$ estudios de la FAO han estimado en la región un área de 39,250 has. aptas para el cultivo de camarón. Además, según MEDEPESCA ${ }^{[7]}$ la región se ha consolidado como un polo de la camaronicultura, gracias a que en sus aguas se encuentran las especies silvestres más conocidas y con mejores resultados de producción, Penneus vannamei y Penneus stylirostris. Esto ha generado muchas expectativas en cuanto a lograr un empuje a la macroeconomía del país. Para el año 2006, Martínez ${ }^{[6]}$, reporta la existencia de 13,600 hectáreas construidas y en producción, y 3000 hectáreas autorizadas para construcción; para ese mismo año se produjo 17 millones de libras cola de camarón con un ingreso bruto de unos 32 millones de dólares.

*Autor para correspondencia: davilaprado@gmail.com
En contraste, la preocupación por alcanzar el desarrollo sostenible; es decir, conciliar el crecimiento económico y la protección del ambiente ${ }^{[4]}$, ha despertado interés por identificar los impactos ambientales de las actividades de desarrollo económico. Por ello, recientemente se discute mucho sobre los posibles impactos ambientales de la camaronicultura ${ }^{[2]}$. Esta actividad está incluida en la lista taxativa de proyectos sometidos por la legislación nacional a un estudio de impacto ambiental[ ${ }^{[1]}$. Esta investigación trata de identificar y caracterizar los principales impactos ambientales que nos ayudan a señalar aquellos puntos críticos en la evaluación de los impactos ambientales de la camaronicultura. Para ello, se realizaron visitas a empresas involucradas, en las distintas etapas del proceso productivo: laboratorios de producción de larvas, granjas camaroneras en fase de operación que usan sistemas semi intensivo e intensivos, y granjas abandonadas.

${ }^{1}$ Decreto 45-94. Reglamento de Permiso y Evaluación de Impacto Ambiental. Articulo 5, inciso c. También incluida más recientemente en el Decreto 76-2006. Sistema de Evaluación Ambiental. Articulo 17, numeral 6 . Este amplia las actividades que están sujetas a un estudio de impacto ambiental y las organiza en tres categorías según el grado de impacto de las actividades y la prioridad para el país. Hernández, J. (2007). Comunicación personal 


\section{DISEÑO METODOLÓGICO, MATERIALES Y EQUIPO}

Se visitaron tres empresas privadas dedicadas a producción de postlarvas, a producción de preadultos de camarón y una abandonada. El Laboratorio de producción de postlarvas de camarón y la Granja abandonada, se ubican en la comunidad de Las Peñitas, a $21 \mathrm{Km}$. al suroeste de la ciudad de León, y la Granja de producción de juveniles, en la comarca Santa Elena, comunidad de Salinas Grandes, a $23 \mathrm{Km}$. al sureste de la ciudad de León.

Se utilizaron dos técnicas propias de la investigación cualitativa: la entrevista semi-estructurada y la observación in situ ${ }^{[10]}$. Las entrevistas se centraron en temas relacionados con: prácticas de manejo, erosión, sedimentación, impactos sobre la vegetación, destrucción de hábitat, uso de nutrientes, uso de fármacos, uso de sustancias químicas, entorno social, fuentes de trabajo, riesgos de enfermedad, densidades de siembra, tipos y formas de alimentación y tasas de recambio de agua. Las personas entrevistadas fueron los técnicos de campo o de laboratorio en las empresas.

La observación in situ se realizó a través de recorridos por las instalaciones de las empresas visitadas donde se tomaron notas sobre las técnicas utilizadas en el proceso de producción y los factores ambientales afectados: cobertura vegetal, suelo, aguas, procesos ecológicos, flora, fauna y factores culturales.

Los datos fueron registrados en una libreta de campo para luego identificar los impactos ambientales generados en el proceso productivo de la camaronicultura.

En este estudio se utiliza un diagrama de red lineal que identifica las interrelaciones de las acciones causales y los factores ambientales, receptores de los impactos, incluyendo impactos secundarios y terciarios ${ }^{[3]}$.

\section{RESULTADOS Y DISCUSIÓN}

\subsection{DESCRIPCIÓN DEL PROCESO PRODUCTIVO}

El proceso productivo empieza con la producción de postlarvas en el laboratorio, luego en la operación de engorde se siembran en los estanques de las granjas encargadas de la producción de camarones preadultos. Además se debe considerar en el proceso productivo, el abandono de granjas camaroneras. Enmarcándonos en esta visión del proceso productivo, consideraremos cada una de las empresas visitadas, según su etapa en la cadena productiva.

Producción de postlarvas: El Laboratorio estudiado realiza el levantamiento larvario, desde los primeros estadios larvarios hasta el estadio postlarva 12, apto para el cultivo en las granjas camaroneras. El personal que laboraba en la empresa al momento de esta investigación era de nueve personas. La producción se inicia desinfectando con Hipoclorito de Sodio al $65 \%$ los tanques revestidos de lainer y con una capacidad de 50 toneladas métricas. Luego se hace un lavado con abundante agua y se aplica Tiosulfato de Sodio para neutralizar el Hipoclorito de Sodio. A continuación, con una bomba de cuatro pulgadas de diámetro se bombea agua desde la playa situada a unos $50 \mathrm{~m}$. de las instalaciones, hacia un tanque reservorio, donde se filtra el agua con carbón activado que garantiza una mejor calidad de ella se distribuye a los tanques de producción. Una vez llenos los tanques, se procede a introducir a los individuos después que hayan sido aclimatados.

La alimentación durante el ciclo productivo se basa en fitoplancton y zooplancton producidos en una cámara de cultivo de microalgas y una de Artemia salina, respectivamente. El tipo de alimento formulado (comercial) utilizado depende del estado larvario. Los alimentos tienden a ensuciar y bajar la calidad del agua. Para remediar esta situación se hacen recambios de agua, periódicamente. Los desechos orgánicos e inorgánicos (sustancias químicas utilizadas en el proceso) de la producción de la larva y del fitoplancton y zooplancton utilizados para su alimentación, son eliminados durante los recambios de agua. El proceso finaliza con la cosecha de la larva, lista para la siembra en los estanques de granjas camaroneras. El agua utilizada en el proceso (durante los recambios y la cosecha) es vertida en la playa a través de tuberías y no recibe ningún tratamiento previo.

Operación de Granjas: La granja de engorde visitada tiene ocho años en producción. El personal al momento de esta investigación era de 13 personas. El sistema de producción utilizado es mixto (semi-intensivo e intensivo), lo que exige cierto nivel de tecnificación; infraestructuras, equipos, control zoosanitario y de factores fisico-químicos, alimentación balanceada, etc. Cuenta con 34 ha. en producción, distribuidas en tres estanques, con un aprovechamiento de dos ciclos productivos por año. La estación de bombeo consta de una bomba axial con motor estacionario de 32 pulgadas de diámetro, situada a orillas del estero, donde se abastece de agua.

Un ciclo productivo inicia con la preparación de los estanques, previo a la siembra. En esta etapa se hace un lavado del estanque para remover los sedimentos del ciclo anterior y luego se desinfectan utilizando cloro $(\mathrm{Cl})$ y cal $\left(\mathrm{CaCO}_{3}\right)$. También se utilizan fertilizantes: Urea $(\%$ N) y Completo (\% NPK), para aumentar el fitoplancton y zooplancton como alimento. Después de abastecer de agua los estanques, se siembra con postlarvas y se establece un sistema de alimentación artificial (alimentos formulados vendidos en el comercio), que consiste en 
colocar el alimento en charolas, en determinados sitios del estanque ( 2 a 3 por hectárea) que funcionan como comederos a una razón de 15-20 libras / ha 2 veces al día, al momento de la visita (La cantidad depende del nivel de biomasa existente en el estanque). Según el sistema de producción, existe un régimen de recambio de agua que garantiza la eliminación de depósitos de materia orgánica y de otras sustancias inorgánicas presentes en el estanque. El ciclo productivo finaliza con la cosecha del camarón que mejor se comercialice. Durante los recambios de agua y la cosecha, el agua que sale del proceso se vierte en el estero, que será utilizado como suministro de agua en el próximo ciclo productivo (según la capacidad de recambio del estero).

Figura 1. Diagrama de red lineal de los principales impactos ambientales del proceso productivo de la camaronicultura en el Pacífico de Nicaragua
Granja en abandono: La granja visitada, al tiempo de realizar esta investigación, tenía tres años de abandono. En su momento presentaba problemas legales con relación a la propiedad y con la banca. La extensión aproximada que ocupaba era de 20 ha. distribuidas en cuatro estanques. La infraestructura está deteriorada, los muros y el canal reservorio dañados por la erosión y las compuertas destruidas. Con el abandono, los playones están siendo colonizados por la vegetación herbácea.

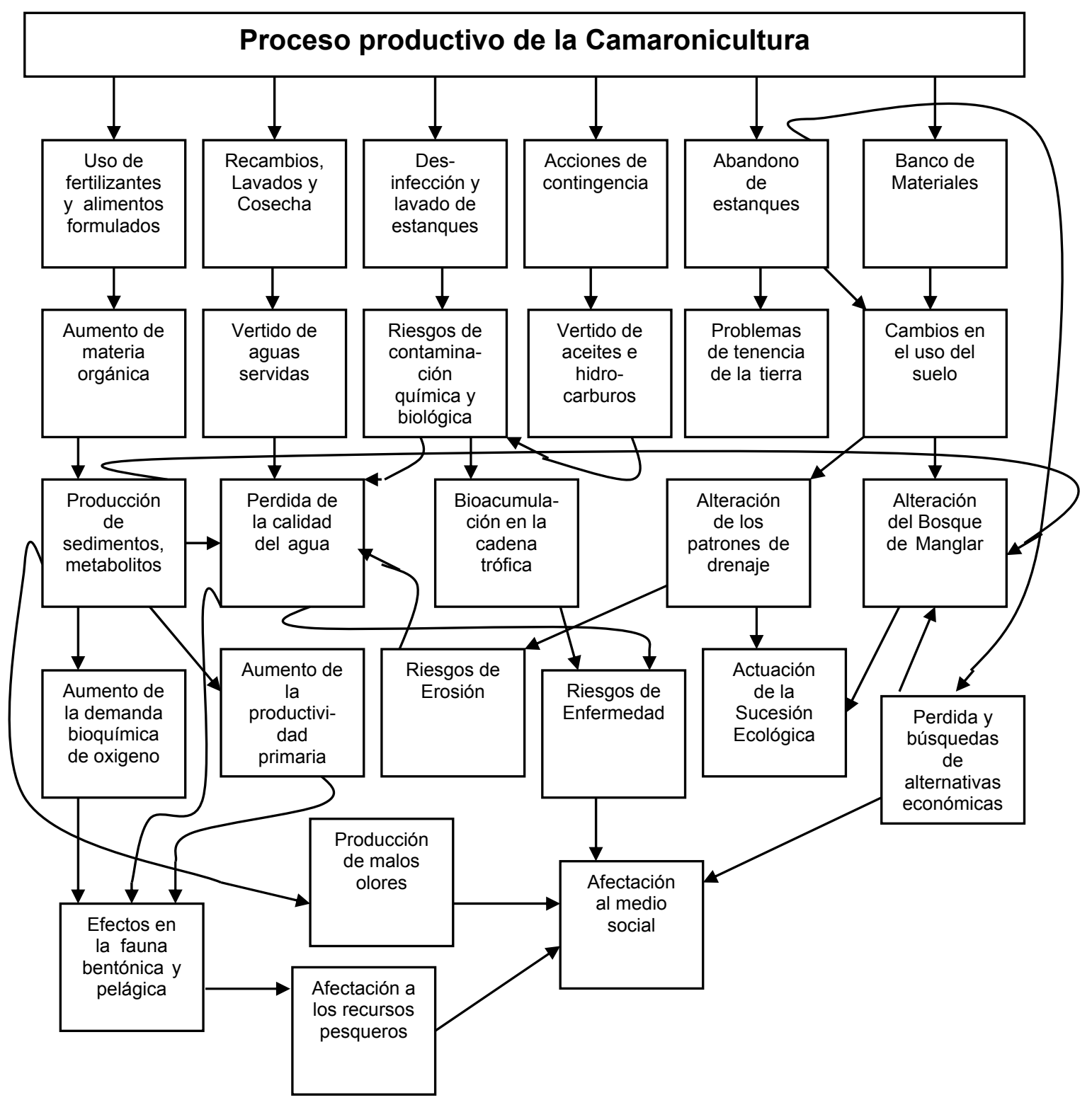




\section{PRINCIPALES IMPACTOS AMBIENTALES}

Los grados de impacto ambiental están directamente relacionados con el sistema de producción implementado [6], los sistemas de producción que se utilizan en Nicaragua son: artesanal, extensivo, semi intensivo e intensivo; a medida que se intensifica el sistema, mayor cantidad de insumos y materia primas son utilizados. Se han identificado actividades comunes en el proceso productivo de la camaronicultura, entre estas tenemos: uso de alimentos naturales y formulados, tasas de recambios periódicos de agua, desinfección y lavados de estanques de producción, cosecha, estanques abandonados, banco de materiales y acciones contingentes. Todas estas actividades tienen un impacto negativo y directo en el agua, suelo y vegetación (Fig. 1).

Encabezan el diagrama las acciones del proceso productivo, las flechas señalan los impactos que se describen consecutivamente como primarios, secundarios y terciarios.

El vertido de aguas servidas de estanques camaroneros en exceso a los esteros con poco recambio puede provocar uno de los impactos más significativos. Dependiendo de la cantidad de residuos orgánicos y sedimentos eliminados, podrían afectar directamente la calidad del agua. Esta situación puede aumentar los sólidos disueltos en el agua y de fitoplancton que en su punto extremo disminuyen la penetración de la luz en el medio acuático de los esteros. Por otro lado, en este escenario extremo, la demanda de oxigeno tiende a aumentar y podría llegar a producir escasez de este recurso con efectos desastrosos para la vida en los esteros. Estos sucesos dependen de la magnitud (sistema de producción) y la frecuencia del impacto de la actividades en cuestión. La granja camaronera en operación utiliza el sistema semi-intensivo en el que se tiene una tasa de recambio diario de agua de $15 \%$ del volumen total ${ }^{[11]}$. Esto junto al grado de aprovechamiento de dos ciclos productivos por año, donde en cada uno se vierten al estero los sedimentos, nos informa de la frecuencia y magnitud del impacto; al que podemos caracterizar como negativo, continuo y de alto impacto

Por otro lado, los sedimentos provenientes de los estanques son capaces de producir metabolitos tóxicos como Sulfito de Hierro $\left(\mathrm{H}_{2} \mathrm{~S}\right)$, Hierro Ferroso $\left(\mathrm{Fe}^{2+}\right)$, Nitrito $\left(\mathrm{NO}_{2}^{-}\right)$, Amonio $\left(\mathrm{NH}_{4}^{+}\right)$y Metano $\left(\mathrm{CH}_{4}\right)$ (Saborio y Bravo 2002). Con las observaciones realizadas durante la visita se logró reconocer olores característicos de metabolitos producidos por sedimentos; esto implica un impacto, directo sobre la macro-fauna bentónica que vive en el fondo de los esteros e indirecto sobre el bosque de manglar.
Existen riesgos de contaminación química y biológica en las aguas servidas de algunos laboratorios. En el proceso productivo utilizan Hipoclorito de sodio, Tiosulfato de Sodio y Formalina. Según protocolos para establecimiento de laboratorios de levantamiento larvario, también se usan Ácido-etilen-diaminotetracético (EDTA) y antibióticos ${ }^{[5]}$. Lo mismo sucede en otros casos, debido al uso de cloro $(\mathrm{Cl})$, cal $\left(\mathrm{CaCO}_{3}\right)$, fertilizantes inorgánicos (NPK), antibióticos y por acciones contingentes.

Los impactos de la contaminación química resultan difíciles de predecir; sin embargo, se podría esperar que estos compuestos entren en la cadena trófica con la posible bio-acumulación en eslabones superiores o quizás causen daños a largo plazo, por tratarse de un impacto acumulativo. La contaminación biológica puede incrementar los riesgos de enfermedades para la población de las comunidades cercanas, por lo que puede considerarse un impacto negativo e indirecto sobre el medio social.

Por otra parte, durante las observaciones realizadas se logró registrar la presencia de fugas de aceites e hidrocarburos provenientes de la estación de bombeo, tal acción contingente se ve agravada por la ubicación a orillas del estero (Fig. 2). Este impacto puede ser caracterizado como negativo, moderado por su magnitud y parcial por su extensión.

De estas acciones sobre el agua del estero, es posible que ocurran impactos indirectos sobre el recurso pesquero. La pesca es la actividad que sostiene económicamente a los pobladores de Salinas Grandes y Las Peñitas, caracterizadas como comunidades pesqueras ${ }^{[1]}$. Por tanto, un impacto indirecto sobre el recurso pesquero ocasionado por la contaminación del medio acuático, afectaría significativamente ambas comunidades.

Figura 2. Fuga de aceites e hidrocarburos de la estación de bombeo ubicada a orillas del estero. Se señaliza el sector con una película oscura sobre la superficie del agua del estero. Foto: Elizabeth Ramírez.

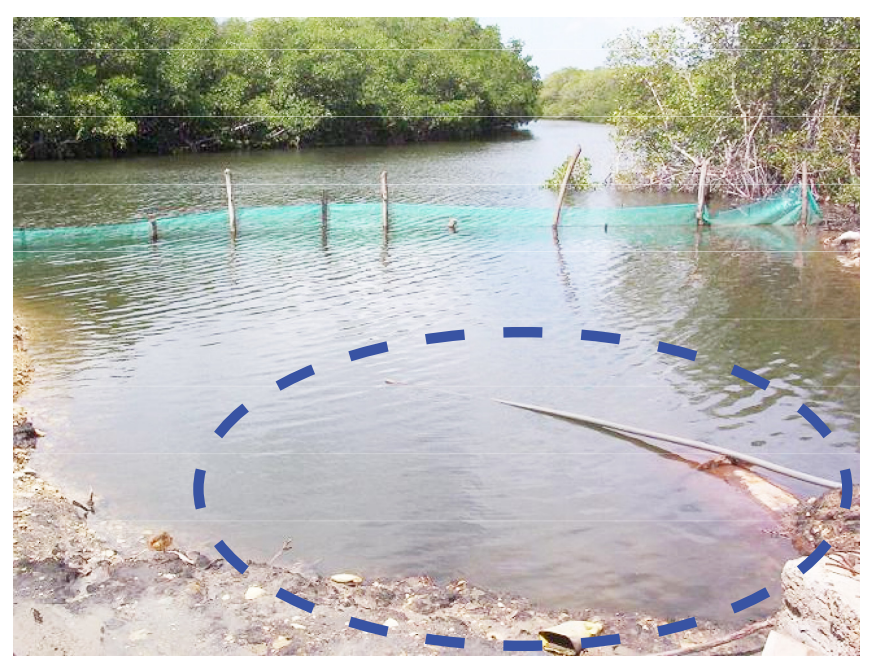


Por otro lado, existe riesgo de enfermedades para los veraneantes. Las Peñitas es un balneario que constituye durante las vacaciones de verano un clásico destino para los habitantes de la ciudad de León. Aunque no se conozca la magnitud de la contaminación de las aguas costeras por los vertidos del laboratorio de producción de larvas, esta situación incrementa la posibilidad de que se produzcan afectaciones a la salud.

Parte de las actividades de la granja en operación, son las de mantenimiento. En estas se insertan las actividades de reparación de muros después de cada ciclo productivo. En la granja se ha iniciado una explotación de banco de materiales (Fig. 3), éste ejerce un impacto directo sobre el suelo y bosque de manglar, y crea posibles cambios en el patrón de drenaje que aumentan los riesgos de erosión.

Figura 3. Banco de materiales utilizado para las labores de mantenimiento. Existe un impacto directo sobre el suelo, subsuelo y bosque de Manglar. Foto: Elizabeth Ramírez

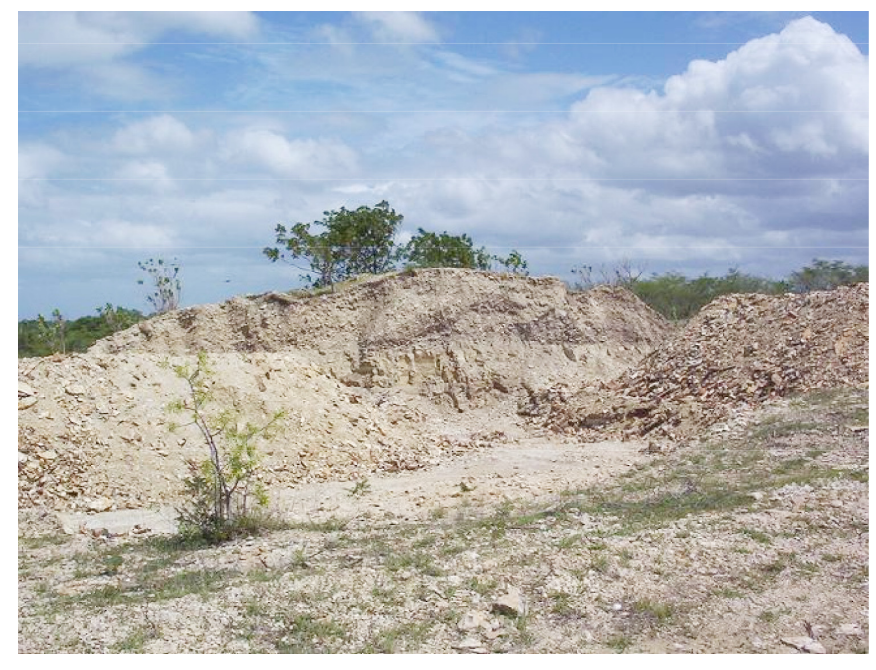

El abandono de granjas camaroneras es otra de las fases del proceso productivo en que se observan impactos en el medio social. Uno de ellos es el desempleo, que crea las circunstancias que ejercen mayor presión sobre los recursos ofrecidos por el ecosistema de manglar. Al no existir alternativas, los pescadores buscan obtener ingresos con la venta de leña de mangle, ocasionando un impacto indirecto sobre este bosque.

Por otro lado, se observa un impacto positivo en la posibilidad de restaurar el área por una etapa seral de la sucesión ecológica. Esto aumentaría la superficie de bosque de manglar y la disponibilidad de hábitat para la vida silvestre. También se genera un problema de propiedad, pues las tierras abandonadas son reclamadas por la comunidad, según los litigios en la administración judicial entre la comunidad y el Banco que ahora figura como dueño de la antigua granja.
Por otra parte, la propiedad está dentro del territorio declarado desde 1983, zona de amortiguamiento del Área Protegida Reserva Natural Isla Juan Venado.

Así que existen riesgos de posibles impactos sobre un área protegida por ley según las decisiones de los involucrados.

\section{CONCLUSIÓN}

El proceso productivo de la camaronicultura puede generar un impacto directo en el medio acuático. La contaminación por descargas de aguas servidas provenientes del proceso productivo semi intensivo e intensivo: producción de larvas, operación y abandono de granjas camaroneras, pueden caracterizarse como un impacto directo, acumulativo sobre las comunidades de organismos acuáticos. La pérdida de la calidad de agua puede resultar un impacto irrecuperable por lo difícil de ejecutar acciones que logren volver al estado inicial de calidad ambiental. Esta caracterización permite señalarlo como el principal punto critico en la evaluación de impacto ambiental de la camaronicultura. Las demás actividades relacionadas con el mantenimiento de las granjas camaroneras ocasionan impactos directos sobre el suelo y bosque de manglar, por cambios en el uso de los suelos. También en este proceso se observan impactos sociales que se generan de manera indirecta de la contaminación de las aguas y su posible impacto sobre el recurso pesquero. Por otro lado, el abandono de granjas camaroneras genera conflictos en el medio social por la tenencia de la tierra y un impacto directo y positivo sobre el bosque de manglar.

\section{AGRADECIMIENTOS}

Agradecemos a las empresas visitadas por el acceso a sus instalaciones y las entrevistas concedidas para la realización de esta investigación.

\section{BIBLIOGRAFÍA}

1.ADPESCA (Administración Nacional de Pesca, NI), (2002), Diagnostico de la actividad pesquera y acuícola. Managua, Nicaragua. 1 disco compacto, $8 \mathrm{~mm}$.

2. Boyd, C.E., (2001). Prácticas de manejo para reducir el impacto ambiental del cultivo de camarón. En Haws, M.C. y Boyd, C.E. eds. Métodos para mejorar la camaronicultura en Centro América. Managua, Nicaragua. Editorial Imprenta. p. 267-295.

\footnotetext{
${ }^{2}$ Alvarado Laguna, A., (2004) Procurador Ambiental, León. Comunicación personal.
} 
3. Canter, Larry W., (1998), Manual de evaluación de impacto ambiental: técnicas para la elaboración de los estudios de impacto ambiental, Madrid, España, 2da edición. Editorial Mc.Graw Hill, p. 99-102

4. Clark, John G., (1995), "Economic development vs. sustainable societies: reflection on the player in a crucial contest", Annu. Rev. Eco. Syst, 26: 225-48

5. Estrada Barcenas, E.A., (2000), Implementación de un sistema de producción de larvas de camarón a escala comercial en el laboratorio de la Estación Biológica Marina Isla Santa Lucía. Tesis (Lic. en Biología). Universidad Nacional Autónoma de Nicaragua, León, Nicaragua, 35 p.

6. Martínez, E., (2007). Acuicultura de camarones marinos L. vannamei en Nicaragua, un enfoque sostenible. Folleto para el componente curricular Acuicultura. Departamento de Biología, UNAN-León. León, Nicaragua. 101 p.

7. Medepesca (Ministerio de Economía Dirección de Promoción y Desarrollo Pesquero, NI), (1997). Anuario pesquero y acuícola. Managua, Nicaragua. p.10,11

8. Ortega, S., (1996). Diagnóstico de las granjas camaroneras del Pacífico de Nicaragua. Informe Técnico. Departamento de Biología, UNAN-León. León, Nicaragua.

9. Otwell, S., Garrido, L., Garrido, V., Benner, R., (2001). Buenas prácticas de acuicultura para la calidad e inocuidad del producto. En Haws, María C. y Boyd, Claude E. eds. Métodos para mejorar la camaronicultura en Centro América. Managua, Nicaragua. Editorial Imprenta. p. 169-231.

10. Rodríguez Gómez, G., Gil Flores, J., García Jiménez, E., (1996). Metodología de la investigación cualitativa. Málaga, España. Ediciones Aljiba. 378 p.

11.Saborio Coze, A., Bravo, J.R., (2002). Manual técnico para el cultivo de camarones marinos en Nicaragua. Managua, Nicaragua. UCA-CIDEA. 50 p. 